\title{
Policy Development for Pandemic Response Using System Dynamics: a Case Study on COVID-19
}

\author{
Charlle Sy ${ }^{1,2}$. Ezekiel Bernardo ${ }^{1}$ - Angelimarie Miguel ${ }^{1}$. Jayne Lois San Juan ${ }^{1,2}$ (D) Andres Philip Mayol $^{3}$. \\ Phoebe Mae Ching ${ }^{4} \cdot$ Alvin Culaba $^{2,3} \cdot$ Aristotle Ubando $^{2,3} \cdot$ Jose Edgar Mutuc $^{1}$
}

Received: 11 April 2020 / Revised: 14 July 2020 / Accepted: 22 July 2020

(C) Springer Nature Singapore Pte Ltd. 2020

\begin{abstract}
The coronavirus disease 2019 (COVID-19) outbreak has burdened several countries. Its high transmissibility and mortality rate have caused devastating impacts on human lives. This has led countries to implement control strategies, such as social distancing, travel bans, and community lockdowns, with varying levels of success. However, a disease outbreak can cause significant economic disruption from business closures and risk avoidance behaviors. This paper raises policy recommendations through a system dynamics modeling approach. The developed model captures relationships, feedbacks, and delays present in a disease transmission system. The dynamics of several policies are analyzed and assessed based on effectiveness in mitigating infection and the resulting economic strain.
\end{abstract}

Keywords COVID-19 · Disease outbreak · Response strategies $\cdot$ Policy recommendation $\cdot$ Impact assessment

\section{Introduction}

The ongoing COVID-19 pandemic is raising unprecedented global health concerns because of its high infection and mortality potential. Since the first case reported on December 30, 2019 , the number of confirmed cases worldwide has grown at an alarming exponential rate (Huang et al. 2020), reaching hundreds of thousands being infected in different countries. As such, the World Health Organization (WHO) declares COVID-19 a public health emergency at an international level ( $\mathrm{Li}$ et al. 2020). This spurred immediate action to identify, prevent, and control the transmission of this virus.

This article is part of the Topical Collection on COVID-19: optimization startegies to combat the pandemic.

Charlle Sy

charlle.sy@dlsu.edu.ph

1 Industrial Engineering Department, De La Salle University, 2401 Taft Avenue, 0922 Manila, Philippines

2 Center for Engineering and Sustainable Development Research, De La Salle University, 2401 Taft Avenue, 0922 Manila, Philippines

3 Mechanical Engineering Department, De La Salle University, 2401 Taft Avenue, 0922 Manila, Philippines

4 Department of Industrial Engineering and Decision Analytics, Hong Kong University of Science and Technology, Clear Water Bay, Kowloon, Hong Kong
Similar precautions and strategies are suggested by experts for combatting COVID-19. Sohrabi et al. (2020) propose immediate diagnosis and isolation for patients with contact to the virus. Xiao and Torok (2020) similarly suggest limiting human-human interaction, identifying and isolating persons with exposure, and providing immediate care to those who have been infected. Globally, each country has put up their own efforts against COVID-19. China, as well as many other countries, imposed travel bans and community lockdowns, especially to and from the epicenter of the outbreak Wuhan, China (Hua and Shaw 2020). Their strict governance has allowed them to "flatten the curve"; however, a second wave is expected because of their premature lifting of the lockdowns and travel ban (Xu and $\mathrm{Li}$ 2020). Nonetheless, late detection allowed their people to travel internationally, leading to a global spread of the virus. Singapore and South Korea, although the latter initially saw a significant spike in cases because of virus transmission during religious gatherings, effectively address this issue through strict screening, contact tracing, and quarantine measures (Gilbert et al. 2020). On the other hand, Europe has become a hotspot for the COVID-19 virus; infection and mortality cases soar in Italy, Spain, Germany, and France, with case counts reaching hundreds of thousands per country. This may be attributed to delayed response, lack of rapid testing, and the high mobility of tourists around Europe (The Lancet 2020). For the same reasons, the United States of America (USA) 
has the most number of cases worldwide, having reached over 300,000 cases as of April 7, 2020 (WHO 2020). In South East Asia, the Philippines has the second most number of cases at 3660 confirmed cases as of April 7, 2020, closely following Malaysia. As a response to COVID-19, the Philippine government implemented a travel ban of foreigners and a gradual implementation of enhanced community quarantine (comparable with lockdowns implemented in other countries). There have also been efforts to increase testing and quarantine capacities (Department of Health 2020). Although vaccination is seen as an effective pharmaceutical-based mitigation strategy, existing vaccines may be ineffectual against novel virus strains (Araz 2013), such as the case in COVID-19. Unfortunately, effective vaccines and treatment for this virus are still under research and development ( $\mathrm{Li}$ et al. 2020).

The persistence of an influenza pandemic can have a significant negative impact on an economy's productive capacity because of its crippling effect on labor supply depending on its morbidity and mortality rate. Fundamental economic theory establishes that labor is required in conjunction with capital and natural resources for the production of valuable goods. Additionally, this triggers a domino effect, wherein the loss in productivity of one sector would inevitably result in the succeeding customer sector to fail to meet their targets and orders. Moreover, government policies, such as school closures and discouraging workers to attend their workplace, can increase worker absenteeism. However, this could be offset by the availability of informal care arrangements and the ability of the parents to work from home (Keogh-Brown 2014). Due to the novelty of the situation, there had yet to be any formal evaluation of the success of online platforms for work in maintaining productivity. Based on the performance of global supply chains, it is apparent that online platforms will fail to sustain the volume of commercial activity prior to the outbreak (Ivanov 2020). Variations in the methods for delivering online education also indicate that universities have yet to converge on a best approach for this scenario, with some universities suspending classes completely and others persisting with online lectures. Prior studies on using technology to deliver the online lectures cite difficulties due to lack of reliable Internet connection, promoting student initiative and autonomy in learning, and the learning delays of adjusting to the technology (Jowsey et al. 2020; Cuaca Dharma et al. 2017). These indicate that, although quarantine is necessary to some extent to control the situation, extended quarantine is not an appropriate solution. Furthermore, despite its unpredictability, there is evidence that suggests externalities such as fear-driven behavioral changes could potentially be the largest contributor to the economic costs of disease outbreaks. From the onset of an outbreak until a considerable period after events stabilize, people are likely to take precautionary actions on top of lockdowns and quarantines imposed, such as avoiding public transportation and international travels, avoiding congregative and entertainment events, and limiting shopping to essentials that would all aggregate to an increase in GDP loss in the first year of about $2 \%$ of GDP (KeoghBrown 2014). Thus, although public health is of highest priority, there is also the need to minimize the negative economic impact of pandemic outbreak responses (Xiao and Torok 2020).

Mathematical and statistical models have been used to gain a better understanding of disease outbreaks and aid in emergency preparedness and response decision-making. These models have been integral in providing insights necessary for developing risk management strategies that minimize the transmission of disease outbreaks and other negative impacts, such as shortages in essential resources and economic declines. One particular methodology, system dynamics (SD) has been employed in disease outbreaks such as influenza. It is a simulation and modeling technique that is established in the application of strategic planning. It is a comprehensive systems perspective approach to map relationships present within complex systems and capturing any nonlinearities, feedback loops, and delays. All of this may provide useful insights towards designing and developing policy interventions (Edaibat et al. 2017). Safarishahrbijari et al. (2017) combined system dynamics with particle filtering approach to predict future influenza outbreaks. Their results outlined the improved accuracy and robustness of the prediction of future influenza outbreak, thus proposing the development of standardized guidelines to control and regulate the potential outbreak. Araz (2013) proposed an integrated framework joining system dynamics with analytic hierarchy process (AHP) a decision-making tool for the assessment of public health strategies in an influenza outbreak capturing multiple stakeholder preferences. The results are aimed to aid the decision-makers on health strategies in the event that an influenza pandemic outbreak. Vincenot and Moriya (2011) adopted an integrated concept of system dynamics and individual-based modeling on the impact of resurgence of influenza pandemic. The results highlighted the behavior of spread over different scenarios outlining the significance of spatial disaggregation during outbreaks.

Currently, no studies have been found in the application of system dynamics on the COVID-19 outbreak. To address this gap, this research is aimed to demonstrate the viability of system dynamics as a framework to understand and develop response strategies for disease pandemics such as COVID-19. The general objective of the study is to develop a system dynamics model of COVID-19 for different scenarios aiming to equip decision-makers with evidence-based judgment in the control of the outbreak.

\section{Model Development}

Alternatives to the extended community quarantine are proposed using the SD framework. Healthcare capacity, 
Fig. 1 Causal loop diagram

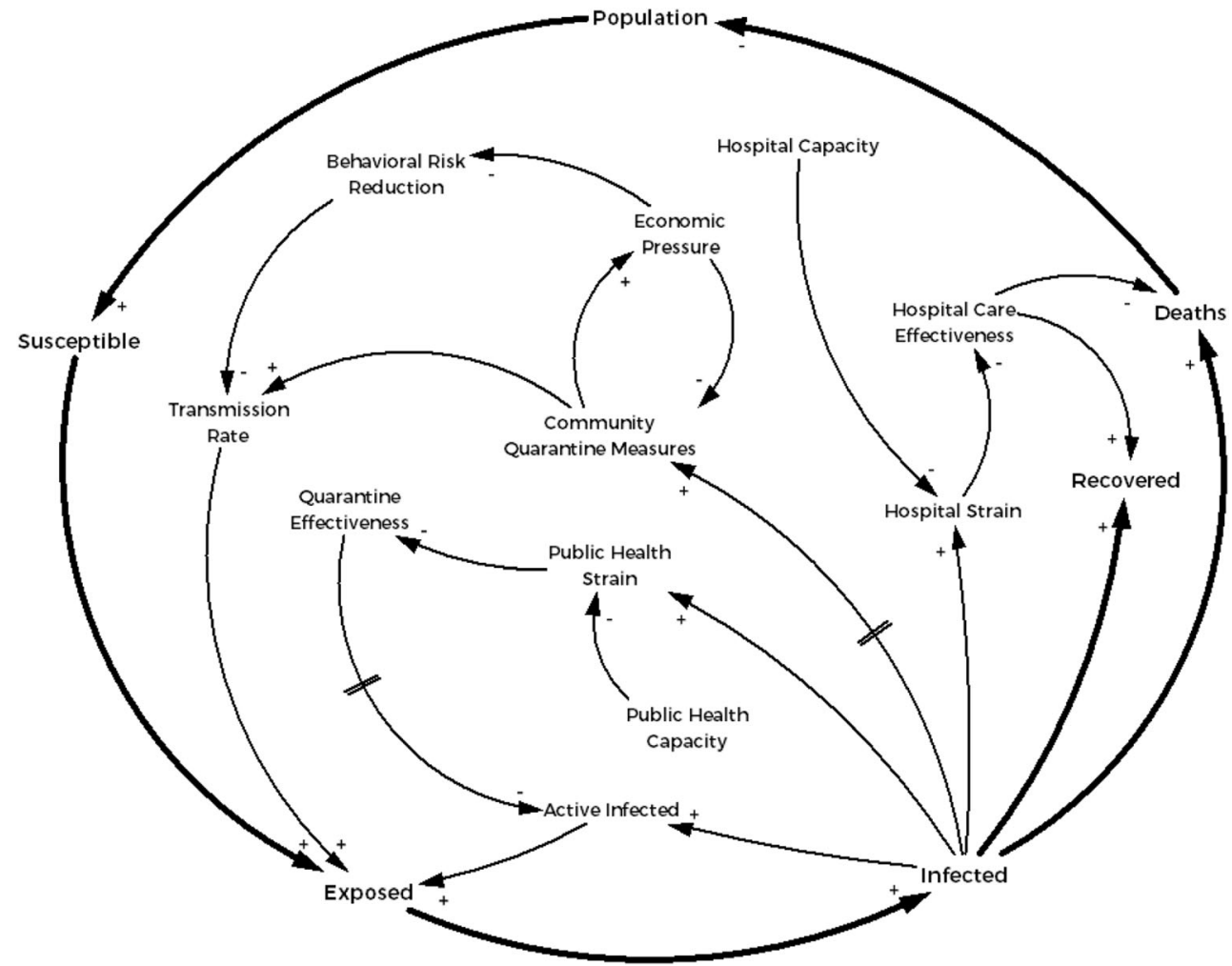

community control policies, and individual human behavior, among the other factors to be considered in this study, vary greatly in the magnitude and nature of their impact to the situation. Yet, it is evident they are critical to pandemic response. Under the SD framework, the relationships between these factors are organized into feedback loops, then are simulated as stocks and flows. However, it is important to note that the numbers or values obtained from SD are not predictive. Instead, SD is used to demonstrate the trend and behavior of problem variables over time as influenced by feedbacks present in the system.

The SD model developed in this study builds upon the basic Susceptible-Infectious-Recovered (SIR) model originally proposed by Grassly and Fraser (2008). Capturing the natural infection process, the number of infected persons, divided into the asymptomatic and symptomatic, will increase the amount of people exposed as shown in the causal loop diagram presented in Fig. 1. The values of these variables are influenced by actions taken to control the situation, such as through the quarantine of those infected, social distancing, travel bans, and personal isolation and protection strategies. Conversely, the magnitude of the problem at various points in time will also influence the magnitude of the response to control the situation. Likewise, a tendency for relaxation is encouraged by negative economic performance. ${ }^{1}$

\footnotetext{
${ }^{1}$ The SD model has been implemented in the software Vensim. This programming language can be acquired from Ventana Systems, Inc. (www.vensim. com), while the model itself is available from the lead author upon request.
}

Hypothetical and literature-based data were used during model implementation, especially because data is not always available and accurate to represent soft variables and highlevel relationships. SD framework was followed, wherein the model is validated against a reference model for the problem variable.

\section{Policy Insights}

The SD model is used to model and simulate the impact of various policies. As a preliminary study, effectiveness will be evaluated in terms of the number of infected people in a population, as this roughly reflects the duration that a country is experiencing a pandemic. Figure $2 \mathrm{a}$ and $\mathrm{b}$ show some of the common responses to the pandemic. The construction of additional hospitals and quarantine centers is one of the primary responses to a pandemic. While some increase in healthcare capacity is necessary, this will only address infection by a limited extent, and is infeasible for developing countries.

Another common policy is to encourage social distancing through either education, publicity, or the closure of establishments (Fig. 2b). This not only reduces the peak levels of infection but also extends the duration that a country is affected by the pandemic. Some consideration may need to be given for the economy or out of necessity, by allowing for certain industries to continue their operations. Even if we yield to economic pressures, overall infection under social distancing 
Fig. 2 Percentage (\%) of population infected vs. time under different policies. a Increase in public health capacity. b Precautions against infection. c Precautions with economic pressure. d Relaxation of precautions for low-risk citizens. e Community quarantine. $\mathbf{f}$ Gradual lifting of quarantine

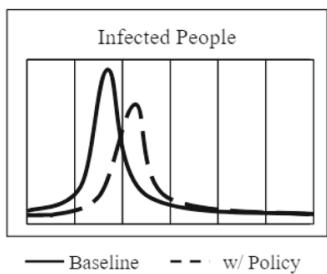

(a) Increase in Public Health Capacity.

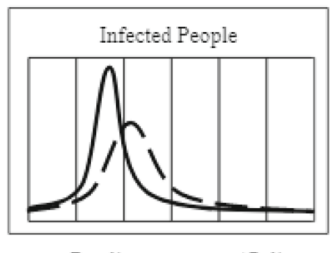

- Baseline - - w/ Policy (d) Relaxation of Precautions for Low-Risk Citizens.

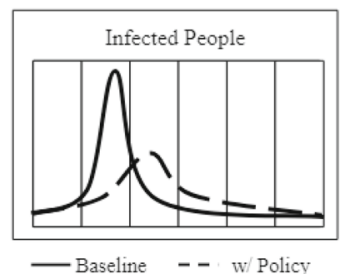

(b) Precautions against Infection.

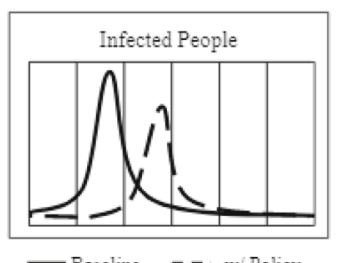

(e) Community Quarantine.

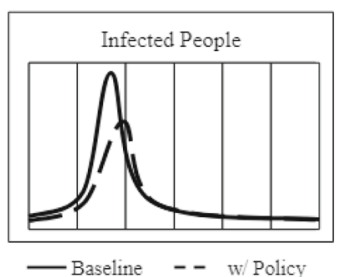

(c) Precautions with Economic Pressure.

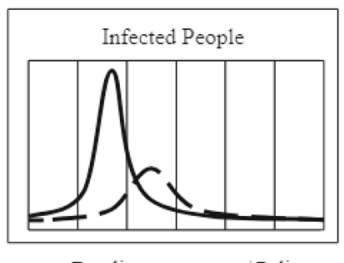

- Baseline - - w/ Policy

(f) Gradual Lifting of Quarantine. will be less than the baseline (Fig. 2c). Similarly, by relaxing the social distancing measures for low-risk demographics, overall infection will still be less than the baseline (Fig. 2d).

Community quarantine is intended to starve the virus, by preventing the spread of the disease between households. Depending on the duration of the quarantine period, this can merely delay the peak of the pandemic without significantly lowering overall infection (Fig. 2e). Gradual quarantine lifting, i.e., reducing the stringency of quarantine over time, gives an opportunity for the economy to recover faster while keeping the potential for infection under control (Fig. 2f).

As a final note, the policies may be implemented together with varying degrees of potency according to the resources and disposition of a particular community.

\section{Policy Recommendations}

The SD framework made it possible to see the interactions between policies in elemental form. This gives the policymaker more room to modify and improve the pre-existing methods for handling pandemics. First, it answers the question of which policies can be implemented together efficiently. Some policies are complementary. Precautionary measures, for instance, will reduce the chance for infection without introducing new consequences to the economy. There are also policies that are inefficient when implemented in basic form, yet have great potential after being modified. In particular, the gradual lifting of quarantine limits infection and also reduces the impairment of the economy resulting from complete quarantines. Rather than investing much in a single policy, multiple policies can leverage on one another to improve the state of a country under the pandemic. From this point, further analysis can be conducted to identify the best parameters for implementation. Impactful parameters include quarantine duration, additional healthcare capacity, and decisions on the quantity and characteristics of low-risk citizens, among other specifics that need to be defined when implementing a policy. By understanding the dynamics of the system, it becomes possible to fine-tune the solution through the parameters, without being too dependent on these values for impact.

\section{Conclusions}

The COVID-19 outbreak has proven to be a complex problem with effects rooted in public health and economics among some. It is crucial that deeper analysis and assessment frameworks are developed to support decision-making for risk management strategies and transmission control interventions that account for the interactions between participating sectors. Based on initial modeling, the most effective strategies focus on avoiding exposure to the virus from happening in the first place; focusing on increasing healthcare capacities only delays the inevitable system collapse as its effectiveness assumes people getting infected first. System dynamics may also be utilized to gain better understanding of other aspects of the system, such as the management of healthcare operations in the face of an influenza pandemic. Furthermore, other modeling techniques, such as optimization modeling, may be employed for resource allocation, especially in the face of scarce essential commodities and medical supplies. It may also be used to select between feasible policy alternatives and to operationalize their implementation based on various, and even conflicting, stakeholder objectives.

\section{Compliance with Ethical Standards}

Conflict of Interest The authors declare that they have no conflicts of interest. 


\section{References}

Araz OM (2013) Integrating complex system dynamics of pandemic influenza with a multi-criteria decision making model for evaluating public health strategies. J Syst Sci Syst Eng 22:319-339. https://doi. org/10.1007/s11518-013-5220-y

Cuaca Dharma HR, Asmarani D, Dewi UP (2017) Basic Japanese grammar and conversation E-learning through Skype and Zoom online application. Procedia Comput Sci 116:267-273. https://doi.org/10. 1016/j.procs.2017.10.055

Department of Health (DOH) (2020) Covid daily testing capacity increases, numbers to improve further to $10 \mathrm{k}$. https://www.doh.gov. $\mathrm{ph} / \mathrm{doh}$-press-release/\%20COVID-DAILY-TESTINGCAPACITY-INCREASES-NUMBERS-TO-IMPROVEFURTHER-TO-10K

Edaibat EA, Dever J, Stuban SM (2017) System dynamics simulation modeling of health information exchange (HIE) adoption and policy intervention: a case study in the state of Maryland. Oper Res Health Care 12:60-70. https://doi.org/10.1016/j.orhc.2017.02.001

Gilbert M, Pullano G, Pinotti F, Valdano E, Poletto C, Boëlle PY, D’Ortenzio E, Yazdanpanah Y, Eholie SP, Altmann M, Gutierrez B, Kraemer MUG, Colizza V (2020) Preparedness and vulnerability of African countries against importations of COVID-19: a modelling study. Lancet 395:871-877. https://doi.org/10.1016/s01406736(20)30411-6

Grassly N, Fraser C (2008) Mathematical models of infectious disease transmission. Nat Rev Microbiol 6:477-487. https://doi.org/10. 1038/nrmicro1845

Hua J, Shaw R (2020) Coronavirus (COVID-19) “Infodemic" and emerging issues through a data lens: the case of China. Int J Environ Res Public Health 17:2309. https://doi.org/10.3390/ijerph17072309

Huang C, Wang Y, Li X, Ren L, Zhao J, Hu Y, Zhang L, Fan G, Xu J, Gu X, Cheng Z, Yu T, Xia J, Wei Y, Wu W, Xie X, Yin W, Li H, Liu M, Xiao Y, Gao H, Guo L, Xie J, Wang G, Jiang R, Gao Z, Jin Q, Wang J, Cao B (2020) Clinical features of patients infected with 2019 novel coronavirus in Wuhan, China. Lancet 395:497-506. https://doi.org/10.1016/S0140-6736(20)30183-5

Ivanov D (2020) Predicting the impacts of epidemic outbreaks on global supply chains: a simulation-based analysis on the coronavirus outbreak (COVID-19/SARS-Cov-2) case. Transp Res E Logist Transp Rev 136:101922. https://doi.org/10.1016/j.tre.2020.101922

Jowsey T, Foster G, Cooper-Ioelu P, Jacobs S (2020) Blended learning via distance in pre-registration nursing education: a scoping review. Nurse Educ Pract 44:102775. https://doi.org/10.1016/j.nepr.2020. 102775

Keogh-Brown M (2014) Macroeconomic effect of infectious disease outbreaks. Ency Health Econ:177-180. https://doi.org/10.1016/b9780-12-375678-7.00608-8

Li X, Wang W, Zhao X, Zai J, Zhao Q, Li Y, Chaillon A (2020) Transmission dynamics and evolutionary history of 2019-nCoV. J Med Virol 92:501-511. https://doi.org/10.1002/jmv.25701

Safarishahrbijari A, Teyhouee A, Waldner C, Liu J, Osgood ND (2017) Predictive accuracy of particle filtering in dynamic models supporting outbreak projections. BMC Infect Dis 17:1-12. https:// doi.org/10.1186/s12879-017-2726-9

Sohrabi C, Alsafi Z, O’Neill N, Khan M, Kerwan A, al-Jabir A, Iosifidis C, Agha R (2020) World Health Organization declares global emergency: a review of the 2019 novel coronavirus (COVID-19). Int J Surg 76:71-76. https://doi.org/10.1016/j.ijsu.2020.02.034

The Lancet (2020) COVID-19: learning from experience. Lancet 395: 1011. https://doi.org/10.1016/s0140-6736(20)30686-3

Vincenot CE, Moriya K (2011) Impact of the topology of metapopulations on the resurgence of epidemics rendered by a new multiscale hybrid modeling approach. Ecol Inform 6:177-186. https://doi.org/ 10.1016/j.ecoinf.2011.04.002

World Health Organization (2020) Coronavirus disease 2019 (COVID19) Situation Report (78). https://www.who.int/docs/default-source/ coronaviruse/situation-reports/20200407-sitrep-78-covid-19.pdf? sfvrsn=bc43e1b_2

Xiao Y, Torok ME (2020) Taking the right measures to control COVID19. The Lancet Infect Dis 20:523-524. https://doi.org/10.1016/ s1473-3099(20)30152-3

Xu S, Li Y (2020) Beware of the second wave of COVID-19. Lancet 395: 1321-1322. https://doi.org/10.1016/S0140-6736(20)30845-X

Publisher's Note Springer Nature remains neutral with regard to jurisdictional claims in published maps and institutional affiliations. 